\title{
Protective Effect of Ethosuximide against Glutamate- Induced Cytotoxicity and Oxidative Stress in C6 Glial Cells
}

\author{
Bilal Sahin \\ Departments of Physiology, Sivas Cumhuriyet University, \\ School of Medicine, Sivas, Turkey \\ Handan Gunes \\ Departments of Physiology, Sivas Cumhuriyet University, \\ School of Medicine, Sivas, Turkey \\ Ziad Joha (Corresponding author) \\ Departments of Pharmacology, Sivas Cumhuriyet University, \\ School of Medicine, Sivas, Turkey \\ E-mail: zead-geha@hotmail.com
}

\begin{abstract}
Purpose: The current study was intended to investigate the potential effect of ethosuximide on glutamate-induced cytotoxicity and oxidative stress in C6 glial cells.
\end{abstract}

Material and Methods: In our study, the C6 cell line was used. To see how ethosuximide affected glutamate-induced cytotoxicity, four cell groups were created. The control group was without any treatment. Cells in the glutamate group were exposed to $10 \mathrm{mM}$ glutamate for $24 \mathrm{~h}$. Cells in the ethosuximide group were treated with various concentrations $(5,10,20$, and $40 \mu \mathrm{M})$ of ethosuximide for $24 \mathrm{~h}$. Cells in the ethosuximide + glutamate group were pre-treated with various concentrations of ethosuximide for $1 \mathrm{~h}$ and then treated with glutamate for $24 \mathrm{~h}$. The cell viability was measured using an XTT assay.Total oxidant status (TOS) and Total antioxidant status (TAS) in the cells were measured by commercial kits.

Results: Ethosuximide at the concentrations of 10,20 , and $40 \mu \mathrm{M}$ significantly increased the cell viability in C6 cells $(\mathrm{p}<0.001)$. Ethosuximide at the concentration of $40 \mu \mathrm{M}$ significantly decreased the TOS levels $(\mathrm{p}<0.05)$ whereas didn't alter the TAS levels $(\mathrm{p}>0.05)$.

Conclusion: Ethosuximide can cause neuroprotective effect on C6 cells. Additionally, one of the potential mechanisms involved in this effect is inhibition of oxidative stress.

Keywords: Ethosuximide, Oxidative Stress, cell viability, C6 Glioma.

\section{Special Issue of Health Sciences}

DOI: $10.7176 /$ JSTR/7-08-10

\section{Introduction}

Glutamate, the central nervous system's principal excitatory neurotransmitter, is involved in brain development as well as functions like sensory activity, memory, learning, synaptic transmission modulation and movement control [1]. Overstimulation of both ionotropic and metabotropic glutamate receptors, on the other hand, has been linked to neuronal injury in a number of neurodegenerative diseases, including Parkinson's disease, Huntington's disease, dementia complex, amyotrophic lateral sclerosis, and Alzheimer's disease [2]. Neurologic trauma, hypoglycemia, stroke, and epilepsy are examples of acute shocks that cause enormous brain cell death and have been linked to excitatory imbalance [3]. Two key mechanisms of glutamate cytotoxicity have been identified in vitro: excitotoxicity as well as cystine transport inhibition-related oxidative stress [4]. Excessive glutamate 
exposure causes membrane depolarization, which can lead to cell death if left untreated. The oxidative stress process of glutamate cytotoxicity is the result of simultaneous cystine transport blockage and increased reactive oxygen species production. For intracellular glutathione (GSH) synthesis, cysteine is the rate-limiting amino acid substrate. Almost majority of the extracellular cysteine is in the oxidized cystine state due to its redox instability. As a result, extracellular cystine is the main source of intracellular cysteine for GSH biosynthesis. Because glutamate and cystine share the same amino acid transporter, they compete for transport into cells. Cysteine transport is limited in the presence of high extracellular glutamate levels, resulting in cellular GSH depletion. When GSH, the main cellular antioxidant, is depleted, the cell becomes more vulnerable to oxidative damage. Peroxides produced by lipoxygenase and monoamine oxidase action may contribute to oxidative stress, which causes GSHdepleted cells to die. In cell lines such as C6 and PC-12, the development of oxidative stress by glutamate has been shown to be the predominant cytotoxic pathway. There is considerable evidence that glutamate-induced disruption of intracellular redox homeostasis is a major contributor to cellular damage in vivo [4].

For the treatment of absence seizures, ethosuximide was offered as an effective and relatively welltolerated medicine. The decrease of low-threshold T-type calcium flows in thalamic cells is the principal mechanism of effet of ethosuximide in the treatment of absence seizures [5]. Ethosuximide has been shown to protect against NRHypo (NMDA receptor hypofunction) neurotoxicity [6]. Ethosuximide has been shown to enhance the phenotypes of many neurodegenerative disorder models by modifying the expression of DAF-16/ FOXO target genes [7]. The Kornfeld lab was one of the first to find that ethosuximide treatment extended the lifespan of wild type C. elegans. Following that, ethosuximide was found to rescue $\mathrm{C}$. elegans neurons in three different neurodegenerative models: frontotemporal dementia with parkinsonism-17, amyotrophic lateral sclerosis, and adult-onset neuronal ceroid lipofuscinosis. The neuroprotective benefits of ethosuximide are not restricted to worms; it has also been reported to be effective in a human neuroblastoma cell culture model of Huntington's disease [8]. The neuroprotective benefits of ethosuximide are not restricted to worms; it has also been reported to be effective in a human neuroblastoma cell line model of Huntington's disease [7]. Additionally, ethosuximide reduced neuronal damage and cognitive impairments in amyloid beta toxin-induced Alzheimer's disease model of rat [9], indicating that its protective benefits could be translated to humans and repurposed as a general neuroprotective drug [8]. However, the effect of ethosuximide on glutamate-induced cytotoxicity and the mechanisms behind it remain unknown. The effect of ethosuximide on glutamate-induced cytotoxicity and oxidative stress in C6 glial cells was investigated in this work.

\section{Materials And Methods}

\subsection{Cell Culture}

Because of its glutamate-induced cytotoxicity, the C6 Glioma (CRL107) cell line was chosen in this work [10]. The American Type Culture Collection provided C6 Glioma cell lines. The cells were grown in DMEM (Thermo Fisher Scientific, Altrincham, UK) with 10\% Fetal Bovine Serum (FBS) (Sigma-Aldrich Co., St Louis, MO, USA), 1\% L-glutamine (Sigma-Aldrich Co., St Louis, MO, USA), and $1 \%$ penicillin/streptomycin (Sigma-Aldrich Co., St Louis, MO, USA) (Sigma-Aldrich Co., St Louis, MO, USA). The cells were kept at $37^{\circ} \mathrm{C}$ in a humidified environment containing $5 \% \mathrm{CO} 2$. Ethosuximide (Sigma-Aldrich Co., St Louis, MO, USA) and glutamate (Sigma-Aldrich Co., St Louis, MO, USA) were dissolved in DMEM, and stock solutions were prepared before treatment.

\subsection{Glutamate-induced cytotoxicity}

To test the effect of ethosuximide on glutamate-induced cytotoxicity, four cell groups were created. It was not applied any treatment to the cells in the control groups. Cells in the glutamate group were treated with glutamate at dose of $10 \mathrm{mM}$ and then incubated for $24 \mathrm{~h}$. According to the literature, the glutamate concentration $(10 \mathrm{mM})$ that causes the death of $50 \%$ of glial cells was chosen [11]. ethosuximide was added to cells in the ethosuximide group at different concentrations $(5,10,20$, and $40 \mu \mathrm{M})$ and then the cells were incubated for 24 hours. Ethosuximide was added to cells in the ethosuximide + glutamate group at different concentrations $(5,10,20$, and $40 \mu \mathrm{M})$ for 1 hours and then $10 \mathrm{mM}$ glutamate was applied for 24 hours.

\subsection{Cell Viability Assay}

The viability of cells were evaluated using the XTT test (Roche Diagnostic, MA, USA). Cells were cultivated in 96 -well plates at the density of $1 \times 104$ cells for each well in $100 \mu$ of DMEM and incubated overnight before ethosuximide. The glutamate-induced cytotoxicity process was carried out

78 | P a g e 
as described above. The medium was withdrawn the next day after 24 hours of incubation, and the wells were cleaned twice with phosphate-buffered saline (PBS). In the final phase, $100 \mu$ of DMEM without phenol red and a mixture of $50 \mu \mathrm{L}$ XTT labeling solution were applied to each well, and the plates were kept at $37^{\circ} \mathrm{C}$ for 4 hours. After shaking the plates, the absorbance was measured at $450 \mathrm{~nm}$ using an ELISA microplate reader (Thermo Fisher Scientific, Altrincham, UK). All of the tests were repeated three times, and cell viability was calculated as a percentage of viable cells in comparison to the control (Figure 1).

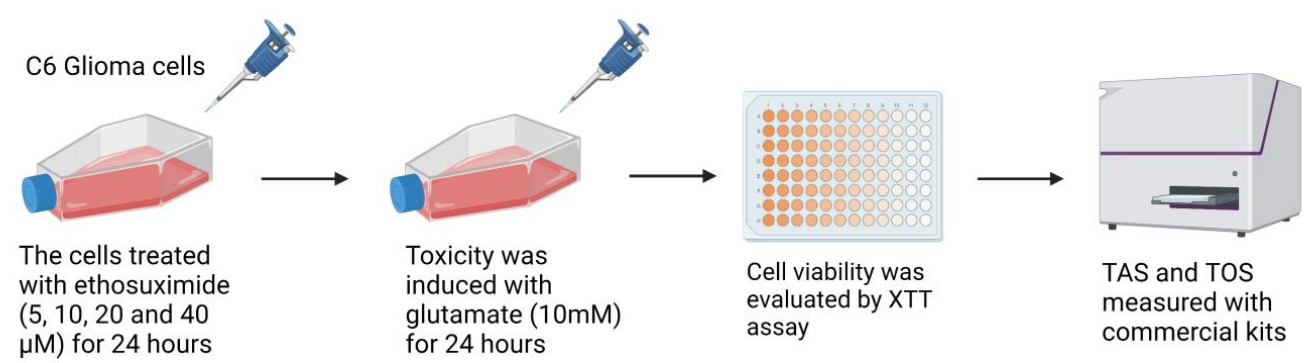

Figure 2 Experimental design of study (created by BioRender).

\subsection{Preparation of cell homogenates}

The cells in each group were collected by sterile tubes. The supernatants were collected after centrifugation at $2000 \mathrm{rpm}$ for about 10 minutes. The cells in the tubes were suspended by diluting the cell suspension with PBS ( $\mathrm{pH}:$ 7.4) to a cell concentration of approximately $1 \mathrm{million} / \mathrm{ml}$. Repeated freeze-thaw cycles were applied to the cells to burst and allow the internal components to escape. They were centrifuged at $4000 \mathrm{rpm}$ for about 10 minutes at a temperature of $4^{\circ} \mathrm{C}$. Then, the supernatants were gathered for analysis of TOS, TAS and total protein levels (Taskiran, \& Ergül, 2021).

\subsection{Total Antioxidant Status And Total Oxidant Status Evaluation}

The levels of TAS and TOS in cell supernatants were measured using an automated test method, which was developed by Erel [13]. The total antioxidant status assay depends on controlling the reaction proportion of free radicals by determining the absorbance of colored dianisidyl radicals throughout free radical reactions starting with the generation of hydroxyl radicals. Antioxidants in the samples should prohibit coloring proportional to their concentrations. The obtained results were expressed in $\mu$ mol Trolox Eq/mg protein. In the total oxidant status, ferrous ions are oxidised to ferric ions when the medium contains enough oxidizers so that the test enables TOS levels to be determined by measuring the level of ferric ions through the use of orange xylenol. To calibrate the assay, hydrogen peroxide was used. The obtained results were expressed at $\mu \mathrm{mol} \mathrm{H} 2 \mathrm{O} 2 \mathrm{Eq} / \mathrm{mg}$ protein. Bradford protein assay kit (Merck Millipore, Darmstadt, Germany) was used to determination of total protein levels in samples.

\subsection{Statistical analysis}

The statistical significance for the tests was found out using one way ANOVA followed by a Tukey post hoc test (SPSS 14.0 for Windows) for multiple comparisons between groups. Data acquired from the cell viability tests were stated as the mean \pm standard error. Significance level was determined as $p$ $<0.05$.

\section{Results}

\subsection{Effect of Ethosuximide at different doses on C6 Cell Viability after cytotoxicity induced by glutamate}

An XTT cell proliferation test was used to assess protective properties of ethosuximide against glutamate-induced cytotoxicity in C6 cells. The effects of various doses of ethosuximide $(5-40 \mu \mathrm{M})$ on cell viability in both control and glutamate-treated $\mathrm{C} 6$ cells were investigated in this work. Ethosuximide at doses of $5,10,20$, and $40 \mu \mathrm{M}$ was added to cells for 1 hour before they were incubated with or without glutamate at dose of $10 \mathrm{mM}$ for the next 24 hours. Figure 2 shows that preincubating C6 cells with glutamate for 24 hours decreased cell viability substantially compared to the control group ( $\mathrm{p}<0.001$; Figure 2). However, as compared to the glutamate-treated group,

79 | P a g e 
ethosuximide at doses of 10, 20, and $40 \mu \mathrm{M}$ improved cell viability $(\mathrm{p}<0.001$; Figure 2$)$. Furthermore, ethosuximide did not affect C6 survival at any dose when compared to the control group ( $p>0.05$; Figure 2).

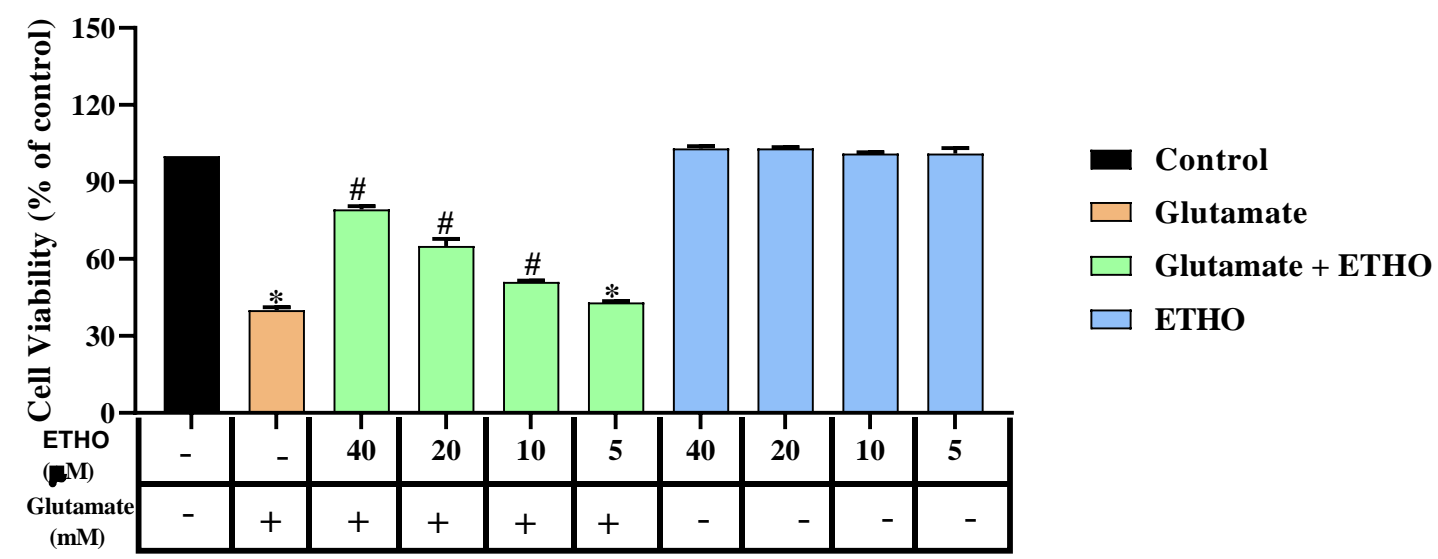

Figure 2. Effect of Ethosuximide on cell survival in C6 cells after glutamate -induced cytotoxicity. The data are expressed as mean \pm standard error mean. ${ }^{*} \mathrm{p}<0.001$ as compared with control group; ${ }^{\#} \mathrm{p}<$ 0.001 as compared with glutamate-treated group.

\subsection{Effect of Ethosuximide on TAS and TOS Levels in C6 cells after cytotoxicity induced by glutamate}

Ethosuximide at dose of $40 \mu \mathrm{M}$ was added to cells for 1 hour before they were incubated with or without glutamate at dose of $10 \mathrm{mM}$ for the next 24 hours. Figure 3 shows that preincubating C6 cells with glutamate for 24 hours decreased TAS levels substantially compared to the control group (p < 0.05; Figure 3A). In comparison to control ( $p>0.05$; Figure 3A) and glutamate-treated groups $(\mathrm{p}>$ 0.05; Figure 3A), ethosuximide did not alter TAS level in C6 cells. On the other hand, preincubating C6 cells with glutamate for 24 hours increased TOS levels, substantially compared to the control group ( $\mathrm{p}<0.05$; Figure 3B). In comparison to the glutamate-treated group ( $\mathrm{p}<0.05$; Figure 3B), ethosuximide substantially decreased TOS level in C6 cells.
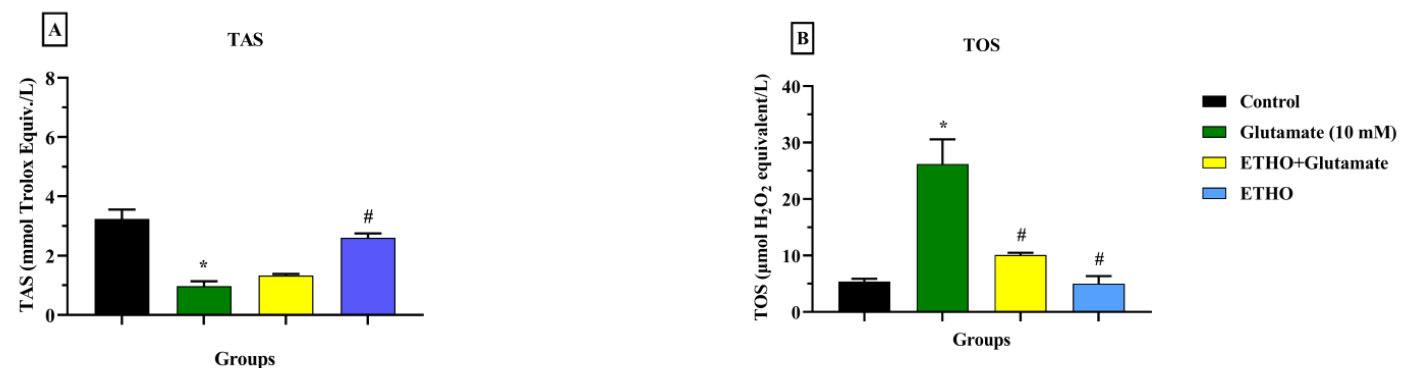

Figure 1. Effect of Ethosuximide on TAS and TOS levels in C6 cells after glutamate -induced cytotoxicity. The data are expressed as mean \pm standard error mean. $* p<0.05$ as compared with control group; ${ }^{\#} \mathrm{p}<0.05$ as compared with glutamate-treated group.

\section{Discussion}

The effects of ethosuximide on glutamate-induced cytotoxicity and oxidative stress in C6 cells were investigated for the first time in this study. It was discovered that pretreatment with ethosuximide reduced deleterious glutamate alterations in C6 cells. In addition, ethosuximide reduced TOS levels but did not change TAS levels after glutamate -induced oxidative stress in the C6 cells. The widespread belief that glutamatergic transmission overactivity causes cell death in a variety of neurological disorders has driven extensive research into medicines that can reduce or eliminate excitotoxicity [3]. Excitotoxicity has been linked to a number of chronic and acute neurological damages, including 
Alzheimer's, Parkinson's, and Huntington's diseases, brain trauma, AIDS dementia complex, stroke [14][15,16] and spinal cord lesion [17] . Excitotoxicity, which is generated by excessive glutamate stimulation and results in excessive calcium ion influx into the cell, is the basic mechanism in the pathogenesis of these disorders. Calcium enters the cell, which causes mitochondrial malfunction and elevates intracellular nitric oxide levels, inducing cell death processes [10].

As cystine and glutamate utilize the same transport pathway, an increase in glutamate in the plasma or extracellular fluids will prevent cystine from being transported. This reduces cysteine availability within the cell for glutathione (GSH) production, resulting in reduced cellular GSH and greater oxidative stress susceptibility. The accumulation of intracellular peroxides after glutamate administration could be linked to the cell's concomitant GSH depletion. Under usual conditions, GSH peroxidase, which uses GSH as a substrate, effectively decomposes both organic and inorganic peroxides in cells. However, substantial depletion of cellular GSH stores, such as that seen after glutamate therapy, may limit GSH peroxidase action and lead to peroxide accumulation. As a result, glutamate-induced peroxide accumulation inside the cell was significantly higher in GSH-arrested cells [18].

An imbalance between oxidants and antioxidant defense mechanisms is known as oxidative stress. This imbalance results in excessive ROS generation, which damages tissues and disrupts the organism's physiological function. Furthermore, research suggests that oxidative stress is a key factor in the development of neurodegenerative illnesses and CNS disorders [12]. In agreement with these information, glutamate reduced cell viability and increased oxidative stress in our research.

Ethosuximide is an antiepileptic medicine used to treat absence epilepsy [19]. Other neuropsychiatric illnesses may benefit from its use as a treatment [20]. It was demonstrated that ethosuximide stimulated proliferation and neuronal differentiation of neural stem cells (NSCs) obtained from the rat hippocampus in vitro, enhanced hippocampal neurogenesis in adult rats, and reversed hippocampal NSCs loss as well as learning and memory problems in the rat model of Alzheimer disease. In addition it was shown that ethosuximide may stimulate neurogenesis via the PI3K/AKT/Wnt/-catenin signaling pathway [9]. In other studies, the $\mathrm{Ca} 2+$ channel blocker ethosuximide and different medicines that lower extracellular and intracellular $\mathrm{Ca} 2+$ levels were found to cause neuroprotective effect [21]. Ttype $\mathrm{Ca} 2+$ channel is one of the voltage-gated $\mathrm{Ca} 2+$ channels that control $\mathrm{Ca} 2+$ levels within cells. In NOD/LtJ mice, it was shown that ethosuximide, a T-type Ca2+ channel blocker, dramatically lowered the increase in age-related ABR (auditory-evoked brainstem response) thresholds, and loss of outer hair cell and spiral ganglion neuron. These effect were linked to the downstream apoptotic pathway [22]. It has been found that inhibiting T-type $\mathrm{Ca} 2+$ channels using ethosuximide can suppress epileptogenesis [23]. In a research on glutamate-induced damage in stroke, $\mathrm{Ca} 2+$ channel inhibition substantially inhibited $\mathrm{Ca} 2+$ entry and postponed neuronal death [23]. Additionally, usual $\mathrm{Ca} 2+$ entry antagonists inhibit neuronal death when applied before and throughout the injury stage of glutamate excitotoxicity [24]. It was reported that calcium channel blockers can inhibit apoptosis or necrosis induced by glutamate [25]. It was found that T-type calcium channel antagonists can reduce calcium homeostasis dysregulation and enhanced vulnerability to rotenone-induced stress in Parkinson's disease [26]. The effects of calcium channel blockers in reducing oxidative stress have been confirmed [27]. Efonidipine, a T-type calcium channel blocker, has been shown to improve renal interstitial fibrosis via activating Nrf2 and subsequently antioxidant enzymes [28]. In agreement with these studies, in this study, it was found that ethosuximide increased cell viability in XTT assay after glutamate-induced cytotoxicity in C6 cells by inhibiting of oxidative stress. In contrast to our findings, Williams et al. demonstrated that ethosuximide did not produce measurable neuroprotection in cultured rat neurons after a range of insults [29]. Given these findings, it's reasonable to hypothesize that ethosuximide can cause therapeutic benefits in neurodegenerative illnesses.

\section{Conclusion}

The results of this investigation indicated that ethosuximide decreased cell death in C6 glial cells after glutamate-induced cytotoxicity.These effects may be a result of oxidative stress inhibition. As a result, ethosuximide may have a neuroprotective effect. However, further research is needed to answer the issues concerning the potential processes involved.

\section{Authors Contributions}

BŞ, ZJ and HG designed and conduct the study and wrote the manuscript. All authors read and approved the manuscript. The authors declare that all data were generated in-house and that no paper mill was used. 


\section{Acknowledgments}

The authors would like to thank the Sivas Cumhuriyet University, School of Medicine, CUTFAM Research Center, Sivas, Turkey, for providing the necessary facilities to conduct this study.

\section{Conflict of Interests}

The authors declare that they have no conflict of interest.

\section{References}

[1] G P Gasic and, Hollmann M. Molecular Neurobiology of Glutamate Receptors. Http://DxDoiOrg/101146/AnnurevPh54030192002451 2003;54:507-36. https://doi.org/ 10. 1146/ ANNUREV.PH.54.030192.002451.

[2] Lipton SA, Rosenberg PA. Excitatory Amino Acids as a Final Common Pathway for Neurologic Disorders. Http://DxDoiOrg/101056/NEJM199403033300907 2010;330:613-22. https://doi. org/10.1056/NEJM199403033300907.

[3] Louzada PR, Lima ACP, Mendonca-Silva DL, Noël F, Mello FG De, Ferreira ST. Taurine prevents the neurotoxicity of $\beta$-amyloid and glutamate receptor agonists: activation of GABA receptors and possible implications for Alzheimer's disease and other neurological disorders. FASEB J 2004;18:511-8. https://doi.org/10.1096/FJ.03-0739COM.

[4] Choi D, Maulucci-Gedde M, Kriegstein A. Glutamate neurotoxicity in cortical cell culture. J Neurosci 1987;7:357-68. https://doi.org/10.1523/JNEUROSCI.07-02-00357.1987.

[5] Glauser TA, Perucca E. Ethosuximide. Treat Epilepsy 2015:460-71. https://doi. org/10.1002/9781118936979.CH34.

[6] Farber NB, Jiang X-P, Heinkel C, Nemmers B. Antiepileptic drugs and agents that inhibit voltage-gated sodium channels prevent NMDA antagonist neurotoxicity. Mol Psychiatry 2002 77 2002;7:726-33. https://doi.org/10.1038/sj.mp.4001087.

[7] Chen X, McCue H V., Wong SQ, Kashyap SS, Kraemer BC, Barclay JW, et al. Ethosuximide ameliorates neurodegenerative disease phenotypes by modulating DAF-16/FOXO target gene expression. Mol Neurodegener 2015101 2015;10:1-14. https://doi.org/10.1186/S13024-0150046-3.

[8] Wong SQ, Pontifex MG, Phelan MM, Pidathala C, Kraemer BC, Barclay JW, et al. $\alpha$-Methyl- $\alpha$ phenylsuccinimide ameliorates neurodegeneration in a C. elegans model of TDP-43 proteinopathy. Neurobiol Dis 2018;118:40-54. https://doi.org/10.1016/J.NBD.2018.06.013.

[9] Tiwari SK, Seth B, Agarwal S, Yadav A, Karmakar M, Gupta SK, et al. Ethosuximide Induces Hippocampal Neurogenesis and Reverses Cognitive Deficits in an Amyloid- $\beta$ Toxin-induced Alzheimer Rat Model via the Phosphatidylinositol 3-Kinase (PI3K)/Akt/Wnt/ $\beta$-Catenin Pathway *. J Biol Chem 2015;290:28540-58. https://doi.org/10.1074/JBC.M115.652586.

[10] Taskiran AS, Ergul M. The effect of salmon calcitonin against glutamate-induced cytotoxicity in the C6 cell line and the roles the inflammatory and nitric oxide pathways play. Metab Brain Dis 2021 2021;1:1-9. https://doi.org/10.1007/S11011-021-00793-6.

[11] Park E, Gim J, Kim DK, Kim C-S, Chun HS. Protective Effects of Alpha-Lipoic Acid on Glutamate-Induced Cytotoxicity in C6 Glioma Cells. Biol Pharm Bull 2019;42:94-102. https://doi.org/10.1248/BPB.B18-00603.

[12] Taskiran, A. Ş., \& Ergül M. The Protective Effect of Hydralazine against Hydrogen Peroxide (H2O2)-Induced Oxidative Damage in C6 Glial Cell Line. Turkish J Sci Heal 2021:124-32. 
[13] Erel O. A novel automated method to measure total antioxidant response against potent free radical reactions. Clin Biochem 2004;37:112-9. https://doi.org/ 10.1016 /j. clinbiochem.2003.10.014.

[14] Cluskey S, Ramsden DB. Mechanisms of neurodegeneration in amyotrophic lateral sclerosis. Mol Pathol 2001;54:386.

[15] Behrens PF, Franz P, Woodman B, Lindenberg KS, Landwehrmeyer GB. Impaired glutamate transport and glutamate-glutamine cycling: downstream effects of the Huntington mutation. Brain 2002;125:1908-22. https://doi.org/10.1093/BRAIN/AWF180.

[16] Mattson MP. Excitotoxic and excitoprotective mechanisms. NeuroMolecular Med 200332 2003;3:65-94. https://doi.org/10.1385/NMM:3:2:65.

[17] Mills CD, Johnson KM, Hulsebosch CE. Role of Group II and Group III Metabotropic Glutamate Receptors in Spinal Cord Injury. Exp Neurol 2002;173:153-67. https:// doi.org/10.1006/EXNR.2001.7828.

[18] Han D, Sen CK, Roy S, Kobayashi MS, Tritschler HJ, Packer L. Protection against glutamateinduced cytotoxicity in C6 glial cells by thiol antioxidants. Https://DoiOrg/101152/ Ajpregu19972735R1771 1997;273. https://doi.org/10.1152/AJPREGU.1997.273.5.R1771.

[19] Gören MZ, Onat F. Ethosuximide: From Bench to Bedside. CNS Drug Rev 2007;13:224-39. https://doi.org/10.1111/J.1527-3458.2007.00009.X.

[20] Kaufman KR. Antiepileptic drugs in the treatment of psychiatric disorders. Epilepsy Behav 2011;21:1-11. https://doi.org/10.1016/J.YEBEH.2011.03.011

[21] Boehmerle W, Huehnchen P, Lee SLL, Harms C, Endres M. TRPV4 inhibition prevents paclitaxel-induced neurotoxicity in preclinical models. Exp Neurol 2018;306:64-75. https://doi.org/10.1016/J.EXPNEUROL.2018.04.014.

[22] Sang L, Zheng T, Min L, Zhang X, Ma X, Entenman S, et al. Otoprotective effects of ethosuximide in NOD/LtJ mice with age-related hearing loss. Int J Mol Med 2017;40:146-54. https://doi.org/10.3892/IJMM.2017.3004.

[23] Becker AJ, Pitsch J, Sochivko D, Opitz T, Staniek M, Chen C-C, et al. Transcriptional Upregulation of Cav3.2 Mediates Epileptogenesis in the Pilocarpine Model of Epilepsy. J Neurosci 2008;28:13341-53. https://doi.org/10.1523/JNEUROSCI.1421-08.2008.

[24] Limbrick DD, Pal S, DeLorenzo RJ. Hippocampal neurons exhibit both persistent Ca2+ influx and impairment of $\mathrm{Ca} 2+$ sequestration/extrusion mechanisms following excitotoxic glutamate exposure. Brain Res 2001;894:56-67. https://doi.org/10.1016/S0006-8993(00)03303-5.

[25] Sadeghnia HR, Kolangikhah M, Asadpour E, Forouzanfar F, Hosseinzadeh H. Berberine protects against glutamate-induced oxidative stress and apoptosis in PC12 and N2a cells. Iran J Basic Med Sci 2017;20:594. https://doi.org/10.22038/IJBMS.2017.8847.

[26] Tabata Y, Imaizumi Y, Sugawara M, Andoh-Noda T, Banno S, Chai MC, et al. T-type Calcium Channels Determine the Vulnerability of Dopaminergic Neurons to Mitochondrial Stress in Familial Parkinson Disease. Stem Cell Reports 2018;11:1171-84. https://doi.org/10.1016/J.STEMCR.2018.09.006.

[27] Nili-Ahmadabadi A, Ali-Heidar F, Ranjbar A, Mousavi L, Ahmadimoghaddam D, LarkiHarchegani A, et al. Protective effect of amlodipine on diazinon-induced changes on oxidative/antioxidant balance in rat hippocampus. Res Pharm Sci 2018;13:368. https://doi.org/10.4103/1735-5362.235164. 
[28] Chung S, Kim S, Kim M, Koh ES, Yoon HE, Kim H-S, et al. T-type calcium channel blocker attenuates unilateral ureteral obstruction-induced renal interstitial fibrosis by activating the Nrf2 antioxidant pathway. Am J Transl Res 2016;8:4574.

[29] Williams AJ, Bautista CC, Chen R-W, Dave JR, Lu X-CM, Tortella FC, et al. Evaluation of Gabapentin and Ethosuximide for Treatment of Acute Nonconvulsive Seizures following Ischemic Brain Injury in Rats. J Pharmacol Exp Ther 2006;318:947-55. https://doi.org/10.1124/JPET.106.105999. 